\title{
Activity of phosphoglycerate mutase and its isoenzymes in serum after acute myocardial infarction
}

\author{
N Durany, E Carballo, J Joseph, J L Bedini, R Bartrons, A M Ballesta, J Carreras
}

\begin{abstract}
Aims/background-In humans there are three phosphoglycerate mutase (PGM, EC 5.4.12.1) isoenzymes (MM, MB and BB) which have similar distribution and developmental pathways to creatine kinase (CK, EC 2.7.3.2) isoenzymes. Total serum PGM activity increases in acute myocardial infarction with the same time course as creatine kinase activity. The present study was undertaken to determine changes in the activity of PGM and its isoenzymes after acute myocardial infarction.
\end{abstract}

Methods-PGM activity was measured spectrophotometrically, by coupling the formation of 2-phosphoglycerate from 3-phosphoglycerate with enolase, pyruvate kinase and lactate dehydrogenase catalysed reactions. Inter- and intra-assay reproducibility was assessed. PGM isoenzyme activities were measured using cellulose acetate electrophoresis.

Results-Total PGM activity in serum was increased in patients with a confirmed diagnosis of acute myocardial infarction. PGM activity peaked 12 to 24 hours after the onset of symptoms and returned to normal values within 48 hours. Electrophoretic analysis of serum from healthy subjects showed a band corresponding to BB-PGM and two other artefactual bands that did not correspond to adenylate kinase. After myocardial infarction, BBPGM activity increased and MB-PGM and MM-PGM could be detected. On immunoblot analysis, normal serum contained an inactive form of MM-PGM with a smaller molecular weight than that of PGM tissue isoenzymes.

Conclusions-Total serum PGM activity increased in patients with acute myocardial infarction, following the same temporal course as creatine kinase activity. The increase in MM-PGM and MB-PGM activities in these patients was not as high as expected. It is suggested that PGM isoenzymes, after release into the blood, undergo postsynthetic, probably proteolytic, transformation.

(F Clin Pathol: Mol Pathol 1996;49:M298-M300)

Keywords: phosphoglycerate mutase, isoenzymes, serum, acute myocardial infarction.

In mammals there are three isoenzymes of phosphoglycerate mutase (PGM, EC 5.4.2.1), which are derived from the homodimeric and heterodimeric combinations of two different subunits (types $\mathrm{M}$ and B). ${ }^{1}$ These enzymes have similar distribution and developmental pathways to creatine kinase (CK, EC 2.7.3.2) isoenzymes. In adult mammals, skeletal muscle and mature sperm cells contain almost exclusively type MM-PGM, whereas the BB isoenzyme is found in most other tissues. All three isoenzymes are present in substantial amounts in myocardial tissue only. ${ }^{1-3}$ The present study was undertaken to determine changes in the activity of PGM and its isoenzymes in serum after acute myocardial infarction.

\section{Methods}

Enzymes and other biochemicals were purchased from either Boehringer (Mannheim, Germany) or Sigma (St Louis, Missouri, USA). PGM activity was measured spectrophotometrically at $30^{\circ} \mathrm{C}$, by coupling the formation of 2-phosphoglycerate from 3phosphoglycerate with enolase, pyruvate kinase and lactate dehydrogenase catalysed reactions. ${ }^{4}$ Intra-assay reproducibility was tested with 15 replicates and interassay reproducibility was demonstrated by duplicating the assay 15 times. For both assays three serum samples with different PGM activities (9 U/l, $30 \mathrm{U} / 1$ and $127 \mathrm{U} / \mathrm{l}$ ) were used. Inter- and intra-assay coefficients of variation were both $<6 \%$ for the samples with the lowest PGM activity and $<2 \%$ for the samples with the highest PGM activity. CK activity was measured at $37^{\circ} \mathrm{C}$ using an optimised spectrophotometric method with reagents from Boehringer Mannheim (CK NAC activated; catalogue number 1040715) on a BM/Hitachi 717 autoanalyser (Boehringer Mannheim). CK-MB was determined by spectrophotometry after immunological inhibition of the $M$ subunit by a specific antibody purchased from Merck (Darmstadt, Germany; reagent numbers 14109, 14110 and 14112). Enzyme activities were expressed as U/1 serum ( 1 unit $=1 \mu \mathrm{mol}$ substrate converted/minute). PGM isoenzymes were analysed by cellulose acetate electrophoresis as described previously. ${ }^{5}$ Cellulose acetate strips (TITAN III ISO-VIS $94 \times 76$ $\mathrm{mm}$; Helena Laboratories, Beaumont, Texas, USA) were pre-soaked in sodium barbital buffer (50 mM, pH 8.6). The samples $(0.5 \mu \mathrm{l})$ were applied by means of a commercial applicator kit (Super CPK; Helena Laboratories) and electrophoresis was performed for $60 \mathrm{~min}$ utes at $4^{\circ} \mathrm{C}$ and 250 volts. PGM isoenzymes were stained using a mixture containing Tris- $\mathrm{HCl}(10 \mathrm{mM}, \mathrm{pH} 8.0), \mathrm{MgSO}_{4}(10 \mathrm{mM})$, 
Table $1 P G M, C K$ and $C K-M B$ values in serum of healthy subjects and patients with acute myocardial infarction

\begin{tabular}{lllllll}
\hline Enzyme (UIl) & Controls $(n=23)$ & \multicolumn{5}{l}{ Time after onset of symptoms (hours) in patients with acute myocardial infarction } \\
\cline { 3 - 7 } & & $0-12(n=14)$ & $12-24(n=10)$ & $24-48(n=11)$ & $48-72(n=13)$ & $72-96(n=10)$ \\
\hline \multirow{2}{*}{ PGM } & $13.7(5.2)$ & $55.1(20.0)$ & $95.7(22)$ & $49.9(15.9)$ & $28.5(10.5)$ & $22.9(6.2)$ \\
& $(10.8-16.6)$ & $(43.6-66.7)$ & $(79.9-112)$ & $(39.3-60.6)$ & $(22.1-34.8)$ & $(18.4-27.4)$ \\
CK & $90(30)$ & $980(878)$ & $2875(1109)$ & $1667(1074)$ & $758(565)$ & $251(137)$ \\
& $(73-107)$ & $(352-1608)$ & $(2082-3668)$ & $(899-2435)$ & $(286-1230)$ & $(108-395)$ \\
CK-MB & $11.5(2.3)$ & $82.4(68.1)$ & $213(110)$ & $98(57)$ & $43.8(23.9)$ & $27.1(10.9)$ \\
& $(10.2-13.1)$ & $(37.0-128.8)$ & $(134-292)$ & $(57-140)$ & $(28.8-63.0)$ & $(17.0-37.0)$ \\
\hline
\end{tabular}

Results are expressed as mean (SD). $95 \%$ confidence intervals are given in parentheses.

$\mathrm{KCl}(20 \mathrm{mM})$, AMP (15 mM), ADP (2 mM), $\mathrm{NADP}+(0.5 \mathrm{mM})$, glucose $(11 \mathrm{mM})$, 3phosphoglycerate (5 $\mathrm{mM}), 2,3$-bisphosphoglycerate $(50 \mu \mathrm{M})$, 3-(4,5-dimethylthiazol2-yl)-2,5-diphenyltetrazolium bromide $(2.4$ $\mathrm{mM})$, phenazine methosulphate $(0.13 \mathrm{mM})$, noble agar $(8 \mathrm{mg} / \mathrm{ml})$, enolase $(0.2 \mathrm{U} / \mathrm{ml})$, pyruvate kinase $(0.8 \mathrm{U} / \mathrm{ml})$, hexokinase $(0.5$ $\mathrm{U} / \mathrm{ml}$ ), and glucose 6-phosphate dehydrogenase $(1.4 \mathrm{U} / \mathrm{ml})$. The staining mixture $(11 \mathrm{ml}$, freshly prepared) was quickly placed over the cellulose acetate strip and incubated at $37^{\circ} \mathrm{C}$ for 30 minutes in the dark. The reaction was stopped by washing the strip with $5 \%$ acetic acid, the solid layer of staining mixture was removed and the strip was washed gently. Western blot analysis was performed as described by Castellà et al. ${ }^{6}$ Specific antiserum directed against PGM was a gift from $F$ Climent. To isolate MM-PGM from serum, $0.2 \mathrm{ml}$ of serum was loaded onto a DEAECellulose DE 23 (Whatman, Maidstone, Kent, UK) column $(6 \times 1.5 \mathrm{~cm})$ pre-equilibrated with Tris- $\mathrm{HCl}(20 \mathrm{mM}, \mathrm{pH}$ 7.5) containing EDTA (1 $\mathrm{mM}$ ) and $\beta$-mercaptoethanol (1 $\mathrm{mM}$ ). MM-PGM was eluted with $25 \mathrm{ml}$ equilibrating buffer.

One-way analysis of variance with repeated measurements was used for statistical evaluation. When a significant $p$ value was obtained $(p<0.05)$, the difference between means was determined using the Tukey test.

Serum was obtained from healthy blood donors and from patients with suspected acute myocardial infarction admitted to the Coronary Care Unit of the Hospital Clinic i Provincial, Barcelona. The diagnosis was made on the basis of clinical symptoms and electrocardiographic and enzyme findings. Serum samples were taken at different time intervals (table 1), kept at $-80^{\circ} \mathrm{C}$ and analysed within six days. To test the stability of PGM and its isoenzymes in vitro, 10 serum samples from healthy subjects and 10 samples from patients with acute myocardial infarction were assayed before and after freezing. On statistical analysis (paired $t$ test, $\mathrm{p}=0.2$ ) there was no significant difference in the PGM activities of the fresh and frozen samples. Moreover, no significant differences were observed in the electrophoretic patterns of both sample groups.

\section{Results and Discussion}

As shown in table 1, total PGM activity in serum was significantly increased in patients with a confirmed diagnosis of acute myocardial infarction. PGM activity increased in these patients following the same temporal course as total CK and CK-MB activities. PGM activity peaked 12 to 24 hours after onset of symptoms and returned to normal values within 48 hours.

As shown in fig 1 (lane 1), an extract of human heart prepared as described previously ${ }^{1}$ and analysed by cellulose acetate electrophoresis as a control, produced three bands of PGM activity, equivalent to those found in other mammals. ${ }^{1-3}$ In the serum from healthy subjects (lanes 2-4) only the band corresponding to type BB-PGM was present, but two additional bands were detected: one with an electrophoretic mobility larger than that of type BB-PGM (band I) and another overlapping that of BB-PGM (band II). In a series of experiments in which different components of the staining mixture were omitted, these two additional bands were found to be artefactual and did not correspond to adenylate kinase (data not shown). Band I was observed even when 3-phosphoglycerate, all co-factors and all linking enzymes were omitted. Band II required glucose but not ADP to be observed. Both artefactual bands were detected when serum samples were analysed after extensive dialysis. In all serum samples from patients with acute myocardial infarction (fig 1, lanes 5-13) the band corresponding to BB-PGM increased in size. In most samples from patients with acute myocardial infarction, a band with an electrophoretic mobility equivalent to that of MB-PGM was detected. In those samples with the highest PGM activity a weak band corresponding to MM-PGM was observed. Samples from patients with acute myocardial infarction also produced the artefactual bands seen in the samples from healthy subjects.

It has been reported that the M-PGM subunit is inactivated by oxidation of its sulphydryl groups. ${ }^{17}$ Therefore, total PGM activity and the PGM isoenzyme electrophoretic pattern were determined after treatment of the serum samples with dithiothreitol to reduce the disulphide bonds and reverse inactivation resulting from oxidation of the sulphydryl groups. Incubation of serum samples from controls and patients with dithiothreitol (10 $\mathrm{mM}, 10$ minutes, $30^{\circ} \mathrm{C}$ ) produced a slight increase $(30 \%)$ in total PGM activity, which suggested the presence of some PGM molecules that had been inactivated by oxidation of their sulphydryl groups. However, the electrophoretic patterns of the isoenzymes did not vary greatly after preincubation with dithiothreitol.

To test for the presence of inactivated PGM isoenzymes not susceptible to reactivation by 


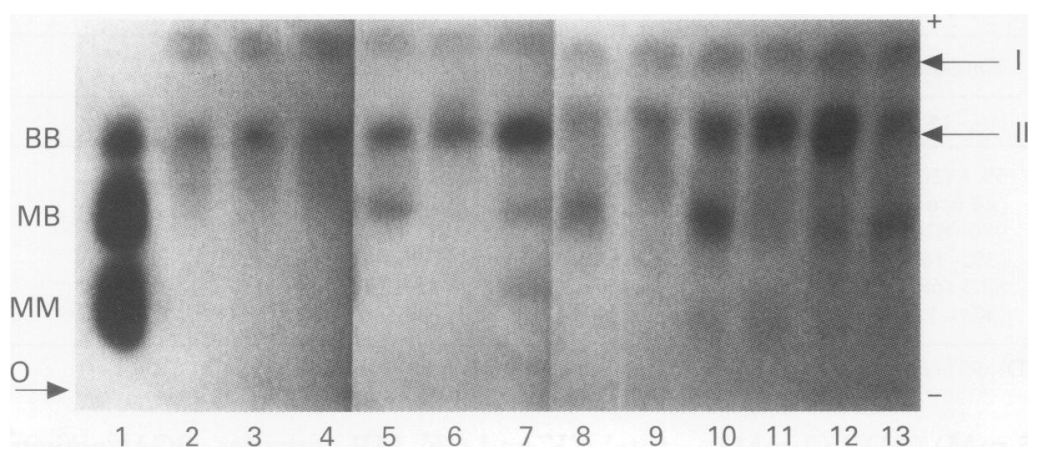

Figure 1 Electrophoretic analysis of PGM isoenzymes in serum samples from healthy subjects (lanes 2-4) and patients with acute myocardial infarction (lanes 5-13). Lane 1, heart extract.

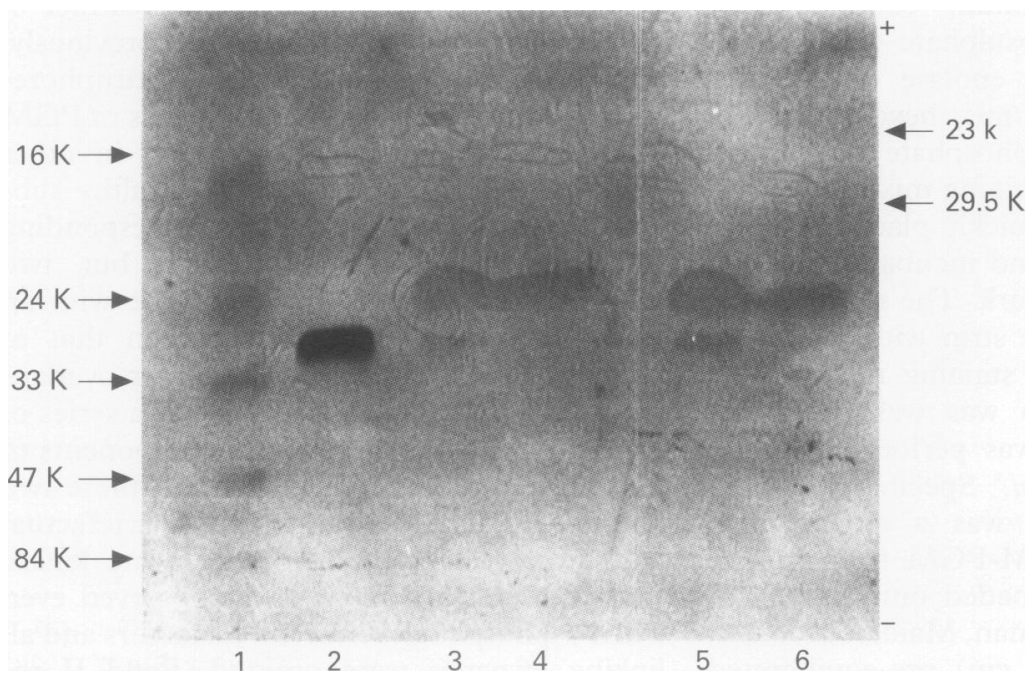

Figure 2 Immunoblot analysis. Lane 1, molecular mass markers; lane 2, muscle extract; lane 3, control serum; lane 4, MM-PGM from control serum; lane 5, serum from a patient with acute myocardial infarction; lane 6, MM-PGM from patient serum.

treatment with dithiothreitol, serum samples were analysed by western blot analysis. Human skeletal muscle extract ${ }^{1}$ was used as a control. As shown in fig 2 , a single band with a molecular mass of 29500 (lane 2) was detected in the muscle extract. The molecular mass of this band is similar to that of PGM subunits from other mammals. ${ }^{1}$ In serum samples from both controls (lane 3) and patients (lane 5) we detected an additional anti-PGM cross reacting protein with lower molecular mass (23 000), which represented most of the anti-PGM cross reacting proteins present in the serum.

To isolate type MM-PGM, serum samples from patients and controls were filtered through a DEAE-Cellulose DE 23 column under conditions preventing the retention of the MM isozyme, ${ }^{8}$ and the flow-through fractions were collected. In agreement with the results of the electrophoretic analysis, only the flow-through fractions eluted from serum from patients with acute myocardial infarction had appreciable PGM activity. But, on immunoblot analysis, the flow-through fractions from both control (fig 2, lane 4) and patient (lane 6) serum cross reacted with anti-PGM serum to give the lower molecular mass band. These results suggest that serum samples contain inactive PGM with a smaller molecular mass $M$ subunit than that of the active PGM isoenzymes. Therefore, we suggest that after release into the blood the M subunit of PGM is inactivated, possibly via a proteolytic process similar to that described for $\mathrm{CK} .{ }^{9}$ As a consequence of the inactivation of the M-PGM subunit, MM-PGM would not be detectable in serum from healthy subjects and the increase in the activities of types MM-PGM and MB-PGM in serum from patients with acute myocardial infarction would not be as high as expected. Inactivation of PGM in this way would also explain the findings in patients with Duchenne muscular dystrophy. It has been reported that in the plasma of these patients both $\mathrm{CK}$ and PGM activities increase, although, in contrast to CK activity, the increase in PGM activity is not as striking. ${ }^{10}$

We thank the staff of the Coronary Care Unit of the Hospital Clinic i Provincial and of the Forensic Institute of Barcelona for their cooperation. This work was supported by FIS grants (numbers 88/1046 and 93/0573).

1 Bartrons R, Carreras J. Purification and characterization of phosphoglycerate mutase isozymes from pig heart Biochim Biophys Acta 1982;708:167-77.

2 Omenn GS, Cheung SCY. Phosphoglycerate mutase isozyme marker for tissue differentiation in man. $A m$ Hum Genet 1974;26:393-9.

3 Mezquita J, Carreras J. Phylogeny and ontogeny of the phosphoglycerate mutases. I. Electrophoretic phenotypes of the glycerate- $2,3-\mathrm{P}_{2}$ dependent phosphoglycerate muof the glycerate-2,3- $\mathrm{P}_{2}$ dependent phosphoglycerate mu tase in $237-45$.

4 Beutler E, Stratton G. Monophosphoglyceromutase (MPGM) In: Red cell metabolism New York: Grune and Stratton, 1975:56-8.

5 Andrés V, Cussó R, Carreras J. Distribution and developmental transition of phosphoglycerate mutase and creatin phosphokinase isozymes in rat muscles of differen fibertype composition. Differentiation 1989;41:72-7.

6 Castellá J, Ureña J, Ludevid D, Carreras J, Climent F. Immunological properties of rat phosphoglycerate mutase Immunological properties of rat phosphoglycerate

7 Mezquita J, Bartrons R, Pons G, Carreras J. Phylogeny and ontogeny of the phosphoglycerate mutases. II. Characterization of phosphoglycerate mutase isozymes from vertebrates by their thermal lability and sensitivity to the sulfhy dryl group reagents. Comp Biochem Physiol 1981;70B 247-55.

8 Carreras J, Bartrons R, Bosch J, Pons G. Metabolism of glycerate $2,3-\mathrm{P}_{2}$. I. Distribution of the enzymes involved in the glycerate-2,3- $\mathrm{P}_{2}$ metabolism in pig tissues. Comp Biochem Physiol 1981;70B:477-85.

9 Panteghini M. Serum isoforms of creatine kinase isoenzymes. Clin Biochem 1988;21:211-18.

10 Chown PJ, Barnard EA, Barnard PJ, Liu PKS, Carter ND. Plasma phosphoglycerate mutase as a marker of muscular dystrophy. $\mathcal{F}$ Neurol Sci 1984;65:201-10. 